DOI: 10.2478 /ausm-2014-0020

\title{
On the combinatorics of extensions of preinjective Kronecker modules
}

\author{
István Szöllősi \\ Babeş-Bolyai University, Cluj-Napoca \\ email: szollosi@gmail.com
}

\begin{abstract}
We explore the combinatorial properties of a particular type of extension monoid product of preinjective Kronecker modules. The considered extension monoid product plays an important role in matrix completion problems. We state theorems which characterize this product in both implicit and explicit ways and we prove that the conditions given in the definition of the generalized majorization are equivalent with our criteria. Generalized majorization is a purely combinatorial construction introduced by its authors in a different setting.
\end{abstract}

\section{Introduction}

In order to understand the motivation behind our work we need to recall briefly the notion of matrix pencil and the problem of matrix subpencil. Kronecker modules and related notions will be presented in Section 3.

A matrix pencil over a field $\kappa$ is a matrix $A+\lambda B$ where $A, B$ are matrices over $k$ of the same size and $\lambda$ is an indeterminate. Two pencils $A+\lambda B, A^{\prime}+\lambda B^{\prime}$ are strictly equivalent, denoted by $A+\lambda B \sim A^{\prime}+\lambda B^{\prime}$, if and only if there exists invertible, constant $(\lambda$ independent $)$ matrices $P, Q$ such that $P\left(A^{\prime}+\lambda B^{\prime}\right) Q=$ $\mathrm{A}+\lambda \mathrm{B}$.

Every matrix pencil is strictly equivalent to a canonical diagonal form, described by the classical Kronecker invariants, namely the minimal indices for columns, the minimal indices for rows, the finite elementary divisors and the infinite elementary divisors (see [7] for all the details).

2010 Mathematics Subject Classification: 16G20, 05A17, 05A20

Key words and phrases: Kronecker algebra, Kronecker module, extension monoid product, matrix pencil, generalized majorization 
A pencil $A^{\prime}+\lambda B^{\prime}$ is called subpencil of $A+\lambda B$ if and only if there are pencils $A_{12}+\lambda B_{12}, A_{21}+\lambda B_{21}, A_{22}+\lambda B_{22}$ such that

$$
A+\lambda B \sim\left(\begin{array}{cc}
A^{\prime}+\lambda B^{\prime} & A_{12}+\lambda B_{12} \\
A_{21}+\lambda B_{21} & A_{22}+\lambda B_{22}
\end{array}\right) .
$$

In this case we also say that the subpencil can be completed to the bigger pencil.

There is an unsolved challenge in pencil theory with lots of applications in control theory (problems related to pole placement, non-regular feedback, dynamic feedback etc. may be formulated in terms of matrix pencils, for details see [9]). This important open problem can be formulated in the following way: if $A+\lambda B, A^{\prime}+\lambda B^{\prime}$ are pencils over $\mathbb{C}$, find a necessary and sufficient condition in terms of their classical Kronecker invariants for $A^{\prime}+\lambda B^{\prime}$ to be a subpencil of $A+\lambda B$. Also construct the completion pencils $A_{12}+\lambda B_{12}, A_{21}+\lambda B_{21}$, $A_{22}+\lambda B_{22}$.

Han Yang was the first to give a representation theoretical modular approach to the matrix subpencil problem, the connection being detailed in [8]. Also, the Kronecker invariants of a module correspond to the classical Kronecker invariants of the associated pencil. In this way a one-to-one correspondence can be made between isoclasses of Kronecker modules and equivalence classes of matrix pencils (with respect to the strict equivalence relation mentioned earlier). In particular, preinjective Kronecker modules correspond to matrix pencils having only minimal indices for columns. This correspondence between matrix pencils and Kronecker modules allows us to deal with the matrix subpencil problem on a module theoretical level, armed with new tools and insights, in addition to the "classical" approach (linear algebra, matrix theory, combinatorics). The matrix subpencil problem itself can be formulated in a very elegant and succinct way in terms of the extension monoid product of certain Kronecker modules (see [17]).

Particular cases of the matrix subpencil problem were considered by Dodig and Stošić in a series of articles (e.g. [3, 4, 6]). One can see that one of the central notions of their work is the so-called generalized majorization, a generalization of the dominance of partitions (which is a well-known notion in partition combinatorics).

Generalized majorization seems to be inevitable when dealing with pencil completion problems. In this paper we give a module theoretical interpretation of this purely combinatorial construction in the form of a particular extension monoid product, together with equivalent formulations and a simple lineartime algorithm to work with in practice. 
The paper is organized in the following way:

- In Section 2 we recall some elementary notions of partition combinatorics, and also present the notion of generalized majorization. Generalized majorization was introduced in [5] and is intensively studied and used by the authors in dealing with technical difficulties of matrix completion problems (e.g. $[3,4,6])$.

- Section 3 is dedicated to a brief survey of the category of Kronecker modules, presenting in some detail the preinjective (and dually preprojective) Kronecker modules.

- In Section 4 we present the notion of extension monoid product, as it applies in the case of preinjective Kronecker modules. Also, this is the place for our new results: Theorem 6 and Theorem 7 giving an implicit and respectively an explicit combinatorial description, followed by an easy linear-time algorithm. Corollary 2 establishes the link between the extension monoid product of preinjective Kronecker modules and the generalized majorization.

We emphasize that all our new results are valid in a field independent context and can be dualized to preinjective modules in a natural way.

From now on, throughout the paper empty sums are considered to be zero. In case of integers $a$ and $b$, by $\{a, \ldots, b\}$ we mean the set of all integers $x$, such that $a \leq x \leq b$, so if $a>b$, then $\{a, \ldots, b\}=\emptyset$. We will usually denote sequences of integers like $\left(a_{1}, a_{2}, \ldots, a_{n}\right)$. If in a certain sequence or subsequence the index of the first element is strictly greater than the index of the last one, the sequence is regarded as being empty.

\section{Some elementary notions of partition combina- torics}

An integer sequence is a sequence $\boldsymbol{a}=\left(\mathrm{a}_{1}, \mathrm{a}_{2}, \ldots\right)$ of integers, with only finitely many nonzero elements. The largest integer $l \geq 0$ with $a_{l} \neq 0$ is called the length of $\mathbf{a}$, denoted by $\ell(\boldsymbol{a})$ (if $\boldsymbol{a}$ is a sequence consisting only of zeros, then $\ell(\mathbf{a})=0)$. We will not distinguish between integer sequences which differ only in the number of zero elements after the $l^{\text {th }}$ position, therefore we regard $\left(a_{1}, a_{2}, \ldots, a_{l}\right),\left(a_{1}, a_{2}, \ldots, a_{l}, 0\right),\left(a_{1}, a_{2}, \ldots, a_{l}, 0, \ldots, 0\right)$ and $\left(a_{1}, a_{2}, \ldots, a_{l}, 0, \ldots\right)$ as being the same integer sequence. Clearly, $\boldsymbol{a} \in \mathbb{Z}^{\mathfrak{n}}$ for 
some $\mathrm{n} \geq \max \{\ell(\boldsymbol{a}), 1\}$. The weight of an integer sequence is the sum of its elements, denoted by $|\boldsymbol{a}|=a_{1}+a_{2}+\cdots$.

A raising operator $\mathrm{R}$ is defined in the following way (on the set of integer sequences having length at most $n$ ):

$$
R: \mathbb{Z}^{n} \rightarrow \mathbb{Z}^{n}, \quad R=\prod_{i<j} R_{i j}^{r_{i j}}
$$

where $r_{i j} \in \mathbb{N}$ and $R_{i j}: \mathbb{Z}^{n} \rightarrow \mathbb{Z}^{n}$,

$$
R_{i j}(a)=\left(a_{1}, \ldots, a_{i}+1, a_{i+1}, \ldots, a_{j-1}, a_{j}-1, a_{j+1}, \ldots, a_{n}\right)
$$

for any pair of integers $i$ and $j$ with $1 \leq i<j \leq n$ and any $\boldsymbol{a}=\left(a_{1}, \ldots, a_{n}\right) \in$ $\mathbb{Z}^{n}$. Note that the terms in the product above commute with each other.

If $\boldsymbol{\mu}=\left(\mu_{1}, \mu_{2}, \ldots\right)$ and $\boldsymbol{\lambda}=\left(\lambda_{1}, \lambda_{2}, \ldots\right)$ are integer sequences and $\sum_{i=1}^{k} \mu_{i} \leq$ $\sum_{i=1}^{k} \lambda_{i}$ for all $1 \leq k$, we say that $\mu$ is dominated (or majored) by $\lambda$. The dominance (or majorization) relation is a partial order on the set of integer sequences and is denoted by $\boldsymbol{\mu} \prec \boldsymbol{\lambda}$.

Another natural order on the set of integer sequences is the lexicographical ordering. If $\boldsymbol{\mu} \neq \boldsymbol{\lambda}$ then $\boldsymbol{\lambda}$ is lexicographically strictly greater than $\boldsymbol{\mu}$ if for the smallest $i$ such that $\mu_{i} \neq \lambda_{i}$ one has $\lambda_{i}>\mu_{i}$. The lexicographical order is a total order on the set of integer sequences.

The following two theorems make the connection between raising operators and dominance relation (for proofs see [10]).

Theorem 1 Let $\mathbf{a} \in \mathbb{Z}^{\mathfrak{n}}$ and $\mathrm{R}$ a raising operator. Then $\mathbf{a} \prec \mathrm{Ra}$.

Conversely, we have:

Theorem $\mathbf{2}$ Let $\mathbf{a}, \mathbf{b} \in \mathbb{Z}^{n}$ be such that $\mathbf{a} \prec \mathbf{b}$ and $|\mathbf{a}|=|\mathbf{b}|$. Then there exists a raising operator $\mathbf{R}$ such that $\mathbf{b}=\mathrm{Ra}$.

If the elements of the integer sequence $\boldsymbol{a}$ are weakly ordered and nonnegative (i.e. $a_{1} \geq a_{2} \geq \cdots a_{l} \geq 0$ ), then we call $\boldsymbol{a}$ a partition of $m=|\mathbf{a}|$. Naturally, everything said so far about integer sequences applies in the case of partitions as well. In particular, the dominance (or majorization) relation is a partial order on the set of partitions. If we denote by $\mathcal{P}_{m}$ the set of partitions of $\mathrm{m}$, we can put together the two previous theorems in form of the following corollary:

Corollary $\mathbf{1}$ Let $\mathbf{a}, \mathbf{b} \in \mathcal{P}_{\mathrm{m}}$ be two partitions of $\mathrm{m}$. Then $\mathbf{a} \prec \mathbf{b}$ if and only if there is a raising operator $\mathbf{R}$ such that $\mathbf{b}=\mathrm{Ra}$. 
In [5] the authors consider a generalization of the dominance relation, the so-called generalized majorization, defined as follows:

Definition 1 Consider the partitions $\mathbf{d}=\left(\mathrm{d}_{1}, \ldots, \mathrm{d}_{\mathrm{x}}\right), \boldsymbol{a}=\left(\mathrm{a}_{1}, \ldots, \mathrm{a}_{\mathrm{y}}\right)$ and $\mathbf{g}=\left(g_{1}, \ldots, g_{x+y}\right)$. Then $\mathbf{g}$ is said to be majorized by $\mathbf{d}$ and $\mathbf{a}$ if the following conditions hold:

$$
\begin{gathered}
d_{i} \geq g_{i+y}, \quad i=1, \ldots x, \\
\sum_{i=1}^{h_{j}} g_{i}-\sum_{i=1}^{h_{j}-j} d_{i} \leq \sum_{i=1}^{j} a_{i}, \quad j=1, \ldots, y, \\
\sum_{i=1}^{x+y} g_{i}=\sum_{i=1}^{x} d_{i}+\sum_{i=1}^{y} a_{i} .
\end{gathered}
$$

Here $h_{j}:=\min \left\{i \mid d_{i-j+1}<g_{i}\right\}, j=1, \ldots, y$. This relation is called the generalized majorization and is denoted in the following way: $\mathbf{g} \prec^{\prime}(\mathbf{d}, \mathbf{a})$.

Remark 1 Observe that in the previous definition we have $0<\mathrm{h}_{1}<\mathrm{h}_{2}<$ $\cdots<\mathrm{h}_{\mathrm{y}}<\mathrm{x}+\mathrm{y}+1$ for the values $\mathrm{h}_{\mathrm{j}}$. Also, this strictly increasing sequence determines another one, denoted by $0<\mathrm{h}_{1}^{\prime}<\mathrm{h}_{2}^{\prime}<\cdots<\mathrm{h}_{\mathrm{x}}^{\prime}<\mathrm{x}+\mathrm{y}+1$, in the following way:

$$
h_{i}^{\prime}=\left\{\begin{array}{ll}
\min \left\{l \in\{1, \ldots, x+y\} \mid l \neq h_{j}, 1 \leq j \leq y\right\} & i=1 \\
\min \left\{l \in\left\{h_{i-1}^{\prime}+1, \ldots, x+y\right\} \mid l \neq h_{j}, 1 \leq j \leq y\right\} & 1<i \leq x
\end{array} .\right.
$$

The elements of these two sequences form disjoint sets, moreover we have $\mathrm{d}_{\mathrm{i}} \geq \mathrm{g}_{\mathrm{h}_{\mathrm{i}}^{\prime}}$ for all $\mathrm{i} \in\left\{1, \ldots, \mathrm{h}_{\mathrm{y}}-\mathrm{y}\right\}$, the sequence $\left(\mathrm{h}_{1}^{\prime}, \mathrm{h}_{2}^{\prime}, \ldots, \mathrm{h}_{\mathrm{h}_{\mathrm{y}}-\mathrm{y}}^{\prime}\right)$ being lexicographically the smallest one with this property. Conversely, if there are sequences $\left(\mathrm{h}_{1}^{\prime}, \mathrm{h}_{2}^{\prime}, \ldots, \mathrm{h}_{\mathrm{x}}^{\prime}\right)$ satisfying $\mathrm{d}_{\mathrm{i}} \geq \mathrm{g}_{\mathrm{h}_{\mathrm{i}}^{\prime}}$ for all $\mathrm{i} \in\{1, \ldots, \mathrm{x}\}$ we can define the sequence $\left(\mathrm{h}_{1}, \mathrm{~h}_{2}, \ldots, \mathrm{h}_{\mathrm{y}}\right)$ in terms of lexicographically the smallest such sequence $\left(\mathrm{h}_{1}^{\prime}, \mathrm{h}_{2}^{\prime}, \ldots, \mathrm{h}_{\mathrm{x}}^{\prime}\right)$ in the following way:

$$
h_{i}=\left\{\begin{array}{ll}
\min \left\{l \in\{1, \ldots, x+y\} \mid l \neq h_{j}^{\prime}, 1 \leq j \leq x\right\} & i=1 \\
\min \left\{l \in\left\{h_{i-1}+1, \ldots, x+y\right\} \mid l \neq h_{j}^{\prime}, 1 \leq j \leq x\right\} & 1<i \leq y
\end{array},\right.
$$

then we get back exactly the sequence $\left(\mathrm{h}_{1}, \mathrm{~h}_{2}, \ldots, \mathrm{h}_{\mathrm{y}}\right)$ given in Definition 1.

\section{The category of Kronecker modules}

In this section we present a short compilation of definitions and well-known facts about the category of Kronecker modules, with emphasis on preinjective 
(and dually preprojective) Kronecker modules. The calculations, justifications and proofs leading to these results can be found in many standard textbooks on representation theory of algebras (e.g. [1, 2, 12, 13]).

Let $\mathrm{K}$ be the Kronecker quiver

$$
K: 1 \underset{\beta}{\longleftarrow} 2
$$

and $\mathrm{k}$ an arbitrary field. The path algebra of the Kronecker quiver is the Kronecker algebra and we will denote it by $\mathrm{kK}$. A finite dimensional right module over the Kronecker algebra is called a Kronecker module. We denote by mod-kK the category of finite dimensional right modules over the Kronecker algebra.

A (finite dimensional) $\mathrm{K}$-linear representation of the quiver $\mathrm{K}$ is a quadruple $M=\left(V_{1}, V_{2} ; \varphi_{\alpha}, \varphi_{\beta}\right)$ where $V_{1}, V_{2}$ are finite dimensional $K$-vector spaces (corresponding to the vertices) and $\varphi_{\alpha}, \varphi_{\beta}: V_{2} \rightarrow V_{1}$ are $\kappa$-linear maps (corresponding to the arrows). Thus a $\mathrm{k}$-linear representation of $\mathrm{K}$ associates vector spaces to the vertices and compatible $\mathrm{k}$-linear functions (or equivalently, matrices) to the arrows. Let us denote by rep- $\mathrm{kK}$ the category of finite dimensional K-representations of the Kronecker quiver. There is a well-known equivalence of categories between mod-kK and rep- $\mathrm{KK}$, so that every Kronecker module can be identified with a representation of $\mathrm{K}$.

The simple Kronecker modules (up to isomorphism) are

$$
S_{1}: K \leftleftarrows 0 \text { and } S_{2}: 0 \leftleftarrows \kappa \text {. }
$$

For a Kronecker module $M$ we denote by $\underline{\operatorname{dim} M}$ its dimension and by $[M]$ the isomorphism class of $M$. The dimension of $M$ is a vector

$$
\underline{\operatorname{dim} M}=\left((\operatorname{dim} M)_{1},(\operatorname{dim} M)_{2}\right)=\left(m_{S_{1}}(M), m_{S_{2}}(M)\right),
$$

where $m_{S_{i}}(M)$ is the number of factors isomorphic with the simple module $S_{i}$ in a composition series of $M, i=\overline{1,2}$. As a representation $M: V_{1} \underset{\varphi_{\beta}}{\stackrel{\varphi_{\alpha}}{\rightleftarrows}} V_{2}$, we have that $\underline{\operatorname{dim} M}=\left(\operatorname{dim}_{\kappa} V_{1}, \operatorname{dim}_{\kappa} V_{2}\right)$.

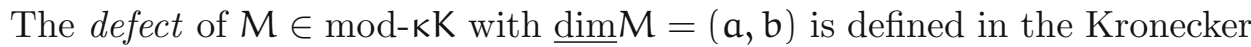
case as $\partial M=b-a$.

An indecomposable module $M \in$ mod-kK is a member in one of the following three families: preprojectives, preinjectives and regulars. In what follows we give some details on the first two of these families. 
The preprojective indecomposable Kronecker modules are determined up to isomorphism by their dimension vector. For $n \in \mathbb{N}$ we will denote by $P_{n}$ the indecomposable preprojective module of dimension $(n+1, n)$. So $P_{0}$ and $P_{1}$ are the projective indecomposable modules $\left(P_{0}=S_{1}\right.$ being simple). It is known that (up to isomorphism) $P_{n}=\left(\kappa^{n+1}, k^{n} ; f, g\right)$, where choosing the canonical basis in $\kappa^{n}$ and $\kappa^{n+1}$, the matrix of $f: \kappa^{n} \rightarrow \kappa^{n+1}$ (respectively of $\left.g: \kappa^{\mathfrak{n}} \rightarrow \mathrm{k}^{\mathfrak{n}+1}\right)$ is $\left(\begin{array}{c}\mathbb{I}_{\mathfrak{n}} \\ 0\end{array}\right)$ (respectively $\left(\begin{array}{c}0 \\ \mathbb{I}_{\mathfrak{n}}\end{array}\right)$ ). Thus in this case

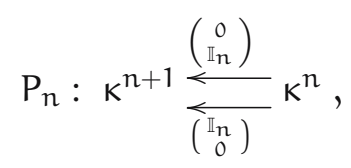

where $\mathbb{I}_{n}$ is the identity matrix. We have for the defect $\partial P_{n}=-1$.

We define a preprojective Kronecker module $\mathrm{P}$ as being a direct sum of indecomposable preprojective modules: $\mathrm{P}=\mathrm{P}_{\mathrm{a}_{1}} \oplus \mathrm{P}_{\mathrm{a}_{2}} \oplus \cdots \oplus \mathrm{P}_{\mathrm{a}_{1}}$, where we use the convention that $a_{1} \leq a_{2} \leq \cdots \leq a_{l}$.

The preinjective indecomposable Kronecker modules are also determined up to isomorphism by their dimension vector. For $n \in \mathbb{N}$ we will denote by $I_{n}$ the indecomposable preinjective module of dimension $(n, n+1)$. So $I_{0}$ and $I_{1}$ are the injective indecomposable modules $\left(\mathrm{P}_{0}=\mathrm{S}_{2}\right.$ being simple). It is known that (up to isomorphism) $I_{n}=\left(\kappa^{n}, \kappa^{n+1} ; f, g\right)$, where choosing the canonical basis in $\kappa^{n+1}$ and $\kappa^{n}$, the matrix of $f: \kappa^{n+1} \rightarrow \kappa^{n}$ (respectively of $g: \kappa^{n+1} \rightarrow \kappa^{n}$ ) is $\left(\begin{array}{ll}\mathbb{I}_{n} & 0\end{array}\right)$ (respectively $\left.\left(\begin{array}{ll}0 & \mathbb{I}_{n}\end{array}\right)\right)$. Thus in this case

$$
I_{n}: K^{n} \underset{\left(0 \mathbb{I}_{n}\right)}{\stackrel{\left(\mathbb{I}_{n} 0\right)}{\longleftarrow}} \kappa^{n+1},
$$

where $\mathbb{I}_{\mathfrak{n}}$ is the identity matrix. We have for the defect $\partial I_{\mathfrak{n}}=1$.

We define a preinjective Kronecker module I as being a direct sum of indecomposable preinjective modules: $\mathrm{I}=\mathrm{I}_{\mathrm{a}_{1}} \oplus \mathrm{I}_{\mathrm{a}_{2}} \oplus \cdots \oplus \mathrm{I}_{\mathrm{a}_{l}}$, where we use the convention that $a_{1} \geq a_{2} \geq \cdots \geq a_{l}$.

The sequence $\left(a_{1}, a_{2}, \ldots, a_{l}\right)$ determines the preinjective (respectively) preprojective Kronecker module up to isomorphism therefore this sequence is called a Kronecker invariant of the module.

The category of Kronecker modules has been extensively studied because the Kronecker algebra is a very important example of a tame hereditary algebra. Moreover, the category has also a geometric interpretation, since it is derived equivalent with the category of coherent sheaves on the projective line. In 
addition, Kronecker modules correspond to matrix pencils in linear algebra, so the Kronecker algebra relates representation theory with numerical linear algebra and matrix theory.

\section{The extension monoid product of preinjective Kro- necker modules}

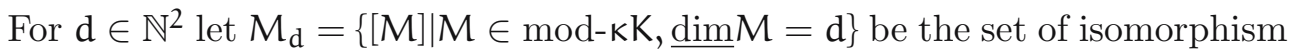
classes of Kronecker modules of dimension d. Following Reineke in [11] for subsets $\mathcal{A} \subset M_{\mathrm{d}}, \mathcal{B} \subset M_{e}$ we define

$\mathcal{A} * \mathcal{B}=\left\{[\mathrm{X}] \in \mathrm{M}_{\mathrm{d}+\mathrm{e}} \mid \exists 0 \rightarrow \mathrm{N} \rightarrow \mathrm{X} \rightarrow \mathrm{M} \rightarrow 0\right.$ exact for some $\left.[\mathrm{M}] \in \mathcal{A},[\mathrm{N}] \in \mathcal{B}\right\}$.

So the product $\mathcal{A} * \mathcal{B}$ is the set of isoclasses of all extensions of modules $M$ with $[\mathrm{M}] \in \mathcal{A}$ by modules $\mathrm{N}$ with $[\mathrm{N}] \in \mathcal{B}$. This is in fact Reineke's extension monoid product using isomorphism classes of modules instead of modules. It is important to know (see [11]) that the product above is associative, i.e. for $\mathcal{A} \subset \mathrm{M}_{\mathrm{d}}, \mathcal{B} \subset \mathrm{M}_{e}, \mathcal{C} \subset \mathrm{M}_{\mathrm{f}}$, we have $(\mathcal{A} * \mathcal{B}) * \mathcal{C}=\mathcal{A} *(\mathcal{B} * \mathcal{C})$. Also $\{[0]\} * \mathcal{A}=\mathcal{A} *\{[0]\}=\mathcal{A}$. We will call the operation "*" simply the extension monoid product.

Remark 2 For $\mathrm{M}, \mathrm{N} \in \bmod -\mathrm{kK}$ and $\mathrm{k}$ finite, the product $\{[\mathrm{M}]\} *\{[\mathrm{~N}]\}$ coincides with the set $\{[\mathrm{M}][\mathrm{N}]\}$ of terms in the Ringel-Hall product $[\mathrm{M}][\mathrm{N}]$ (see Section 4 from [18]).

From now on we deal only with the extension monoid product of preinjective Kronecker modules. It is very important to mention that all results can be dualized in natural way to preprojective Kronecker modules as well.

According to the main result from [16] (Theorem 3.3), the possible middle terms in preprojective (and dually preinjective) short exact sequences do not depend on the base field. This allows us to describe the combinatorial rules governing the extension monoid product of preinjective Kronecker modules in a field independent way. Specifically, this allows us to restate the main result from [18] involving the Ringel-Hall product (valid only over finite fields) in terms of the extension monoid product (in a field independent manner). The following theorem gives an implicit description of the extension monoid product of two arbitrary preinjective Kronecker modules over an arbitrary field: 
Theorem 3 If $a_{1} \geq \cdots a_{p} \geq 0, b_{1} \geq \cdots \geq b_{n} \geq 0$ and $c_{1} \geq \cdots \geq c_{r} \geq 0$ are nonnegative integers, then

$$
\left[\mathrm{I}_{\mathrm{c}_{1}} \oplus \cdots \oplus \mathrm{I}_{\mathrm{c}_{\mathrm{r}}}\right] \in\left\{\left[\mathrm{I}_{\mathrm{a}_{1}} \oplus \cdots \oplus \mathrm{I}_{\mathrm{a}_{\mathrm{p}}}\right]\right\} *\left\{\left[\mathrm{I}_{\mathrm{b}_{1}} \oplus \cdots \oplus \mathrm{I}_{\mathrm{b}_{\mathfrak{n}}}\right]\right\}
$$

if and only if $\mathrm{r}=\mathrm{n}+\mathrm{p}, \exists \beta:\{1, \ldots, \mathrm{n}\} \rightarrow\{1, \ldots, \mathrm{n}+\mathrm{p}\}, \exists \alpha:\{1, \ldots, \mathrm{p}\} \rightarrow$ $\{1, \ldots, n+p\}$ both functions strictly increasing with $\operatorname{Im} \alpha \cap \operatorname{Im} \beta=\emptyset$ and $\exists \mathrm{m}_{j}^{i} \geq$ $0,1 \leq \mathfrak{i} \leq \mathrm{n}, 1 \leq \mathrm{j} \leq \mathrm{p}$, such that $\forall \ell \in\{1, \ldots, \mathrm{n}+\mathrm{p}\}$

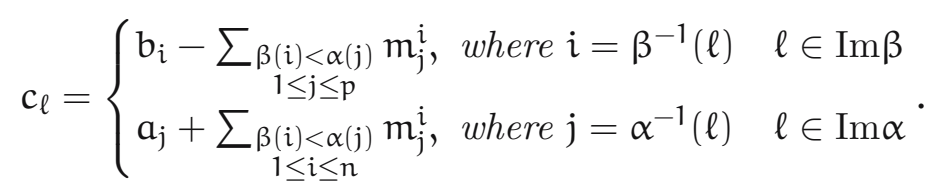

We can formulate another version of the previous theorem, based on Lemma 4 from [19], giving another equivalent characterization of the considered extension monoid product:

Theorem 4 If $a_{1} \geq \ldots a_{p} \geq 0, b_{1} \geq \cdots \geq b_{n} \geq 0$ and $c_{1} \geq \cdots \geq c_{r} \geq 0$ are nonnegative integers, then

$$
\left[\mathrm{I}_{\mathrm{c}_{1}} \oplus \cdots \oplus \mathrm{I}_{\mathrm{c}_{\mathrm{r}}}\right] \in\left\{\left[\mathrm{I}_{\mathrm{a}_{1}} \oplus \cdots \oplus \mathrm{I}_{\mathrm{a}_{\mathrm{p}}}\right]\right\} *\left\{\left[\mathrm{I}_{\mathrm{b}_{1}} \oplus \cdots \oplus \mathrm{I}_{\mathrm{b}_{\mathfrak{n}}}\right]\right\}
$$

if and only if $\mathrm{r}=\mathrm{n}+\mathrm{p}, \sum_{\mathfrak{i}=1}^{n+p} c_{i}=\sum_{i=1}^{p} a_{i}+\sum_{i=1}^{n} b_{i}, \exists \beta:\{1, \ldots, n\} \rightarrow$ $\{1, \ldots, \mathfrak{n}+\mathbf{p}\}, \exists \alpha:\{1, \ldots, p\} \rightarrow\{1, \ldots, n+p\}$ both functions strictly increasing with $\operatorname{Im} \alpha \cap \operatorname{Im} \beta=\emptyset$ such that $\mathrm{b}_{\mathfrak{i}} \geq \mathrm{c}_{\beta(\mathrm{i})}$ and $\mathrm{a}_{\mathrm{j}} \leq \mathrm{c}_{\alpha(\mathrm{j})}$ for $1 \leq \mathrm{i} \leq \mathrm{n}, 1 \leq \mathrm{j} \leq \mathrm{p}$ and for any $j \in\{1, \ldots, p\}$ the following inequality is satisfied:

$$
\sum_{\substack{\beta(i)<\alpha(j) \\ 1 \leq i \leq n}}\left(b_{i}-c_{\beta(i)}\right) \geq \sum_{k=1}^{j}\left(c_{\alpha(k)}-a_{k}\right) .
$$

The following combinatorial rule describes products of the form $\left\{\left[\mathrm{I}_{\mathrm{a}_{n}}\right]\right\} *\left\{\left[\mathrm{I}_{\mathrm{a}_{n-1}}\right]\right\} *$ $\cdots *\left\{\left[\mathrm{I}_{a_{1}}\right]\right\}$ with $0 \leq \mathrm{a}_{\mathrm{n}} \leq \mathrm{a}_{\mathrm{n}-1} \leq \cdots \leq \mathrm{a}_{1}$ increasing. It has been proved in [15] for finite fields and also in [20] (in a field independent context):

Theorem 5 Suppose that $\mathrm{a}_{1} \geq \cdots \geq \mathrm{a}_{\mathrm{n}} \geq 0$ and $\mathrm{c}_{1} \geq \cdots \geq \mathrm{c}_{\mathrm{n}} \geq 0$. Then

$$
\left[\mathrm{I}_{\mathrm{c}_{1}} \oplus \mathrm{I}_{\mathrm{c}_{2}} \oplus \cdots \oplus \mathrm{I}_{\mathrm{c}_{\mathrm{n}}}\right] \in\left\{\left[\mathrm{I}_{\mathrm{a}_{\mathrm{n}}}\right]\right\} *\left\{\left[\mathrm{I}_{\mathrm{a}_{\mathrm{n}-1}}\right]\right\} \cdots *\left\{\left[\mathrm{I}_{\mathrm{a}_{1}}\right]\right\}
$$

if and only if $\sum_{i=1}^{k} c_{i} \leq \sum_{i=1}^{k} a_{i}$ for all $k \in\{1, \ldots, n\}$ with $\sum_{i=1}^{n} c_{i}=\sum_{i=1}^{n} a_{i}$. 
Remark 3 The condition $\sum_{i=1}^{k} c_{i} \leq \sum_{i=1}^{k} a_{i}$ in Theorem 5 says that the partition $\mathbf{c}=\left(\mathrm{c}_{1}, \mathrm{c}_{2}, \ldots, \mathrm{c}_{\mathrm{n}}\right)$ is dominated by $\mathbf{a}=\left(\mathrm{a}_{1}, \mathrm{a}_{2}, \ldots, \mathrm{a}_{\mathrm{n}}\right)$, i.e. $\mathbf{c} \prec \boldsymbol{a}$.

Using Theorem 4 and Theorem 5 we are ready now to prove our first result, which is a characterization of the products of the form

$$
\left\{\left[\mathrm{I}_{\mathrm{a}_{\mathrm{p}}}\right]\right\} *\left\{\left[\mathrm{I}_{\mathrm{a}_{\mathrm{p}-1}}\right]\right\} * \cdots *\left\{\left[\mathrm{I}_{\mathrm{a}_{1}}\right]\right\} *\left\{\left[\mathrm{I}_{\mathrm{b}_{1}}\right]\right\} *\left\{\left[\mathrm{I}_{\mathrm{b}_{2}}\right]\right\} * \cdots *\left\{\left[\mathrm{I}_{\mathrm{b}_{\mathrm{n}}}\right]\right\},
$$

where the integers $0 \leq a_{p} \leq a_{p-1} \leq \cdots \leq a_{1}$ are increasingly ordered, whereas the integers $b_{1} \geq b_{2} \geq \cdots \geq b_{n} \geq 0$ are decreasing.

Theorem 6 If $a_{1} \geq \cdots a_{p} \geq 0, b_{1} \geq \cdots \geq b_{n} \geq 0$ and $c_{1} \geq \cdots \geq c_{r} \geq 0$ are nonnegative integers, then

$\left[\mathrm{I}_{\mathfrak{c}_{1}} \oplus \mathrm{I}_{\mathfrak{c}_{2}} \oplus \cdots \oplus \mathrm{I}_{\mathfrak{c}_{r}}\right] \in\left\{\left[\mathrm{I}_{\mathrm{a}_{\mathrm{p}}}\right]\right\} *\left\{\left[\mathrm{I}_{\mathrm{a}_{\mathrm{p}-1}}\right]\right\} * \cdots *\left\{\left[\mathrm{I}_{\mathrm{a}_{1}}\right]\right\} *\left\{\left[\mathrm{I}_{\mathrm{b}_{1}}\right]\right\} *\left\{\left[\mathrm{I}_{\mathrm{b}_{2}}\right]\right\} * \cdots *\left\{\left[\mathrm{I}_{\mathrm{b}_{\mathfrak{n}}}\right]\right\}$

if and only if $\mathrm{r}=\mathrm{n}+\mathrm{p}, \sum_{i=1}^{\mathrm{n}+\mathrm{p}} \mathrm{c}_{\mathrm{i}}=\sum_{\mathrm{i}=1}^{\mathrm{p}} \mathrm{a}_{\mathrm{i}}+\sum_{i=1}^{n} \mathrm{~b}_{i}, \exists \beta:\{1, \ldots, \mathrm{n}\} \rightarrow$ $\{1, \ldots, n+p\}, \exists \alpha:\{1, \ldots, p\} \rightarrow\{1, \ldots, n+p\}$ both functions strictly increasing with $\operatorname{Im} \alpha \cap \operatorname{Im} \beta=\emptyset$ such that $\mathrm{b}_{\mathrm{i}} \geq \mathrm{c}_{\beta(\mathrm{i})}$ for $1 \leq \mathrm{i} \leq \mathrm{n}$ and for any $\mathrm{j} \in\{1, \ldots, \mathrm{p}\}$ the following inequality is satisfied:

$$
\sum_{\substack{\beta(i)<\alpha(j) \\ 1 \leq i \leq n}}\left(b_{i}-c_{\beta(i)}\right) \geq \sum_{k=1}^{j}\left(c_{\alpha(k)}-a_{k}\right) .
$$

Proof. As a first step, observe that $\left\{\left[\mathrm{I}_{\mathrm{a}_{\mathrm{p}}}\right]\right\} *\left\{\left[\mathrm{I}_{\mathrm{a}_{\mathrm{p}-1}}\right]\right\} * \cdots *\left\{\left[\mathrm{I}_{\mathrm{a}_{1}}\right]\right\} *\left\{\left[\mathrm{I}_{\mathrm{b}_{1}}\right]\right\} *$ $\left\{\left[\mathrm{I}_{\mathrm{b}_{2}}\right]\right\} * \cdots *\left\{\left[\mathrm{I}_{\mathrm{b}_{\mathfrak{n}}}\right]\right\}=\left(\left\{\left[\mathrm{I}_{\mathrm{a}_{\mathrm{p}}}\right]\right\} *\left\{\left[\mathrm{I}_{\mathrm{a}_{\mathrm{p}-1}}\right]\right\} * \cdots *\left\{\left[\mathrm{I}_{\mathrm{a}_{1}}\right]\right\}\right) *\left(\left\{\left[\mathrm{I}_{\mathrm{b}_{1}}\right]\right\} *\left\{\left[\mathrm{I}_{\mathrm{b}_{2}}\right]\right\} * \cdots *\right.$ $\left.\left\{\left[\mathrm{I}_{\mathrm{b}_{n}}\right]\right\}\right)=\left(\left\{\left[\mathrm{I}_{\mathrm{a}_{\mathrm{p}}}\right]\right\} *\left\{\left[\mathrm{I}_{\mathrm{a}_{\mathrm{p}-1}}\right]\right\} * \cdots *\left\{\left[\mathrm{I}_{\mathrm{a}_{1}}\right]\right\}\right) *\left\{\left[\mathrm{I}_{\mathrm{b}_{1}} \oplus \mathrm{I}_{\mathrm{b}_{2}} \oplus \cdots \oplus \mathrm{I}_{\mathrm{b}_{n}}\right]\right\}$. Here we have used the associativity of the extension monoid product and we have applied repeatedly Theorem 3 on the second part of the product to get the equality $\left\{\left[\mathrm{I}_{\mathrm{b}_{1}}\right]\right\} *\left\{\left[\mathrm{I}_{\mathrm{b}_{2}}\right]\right\} * \cdots *\left\{\left[\mathrm{I}_{\mathrm{b}_{\mathrm{n}}}\right]\right\}=\left\{\left[\mathrm{I}_{\mathrm{b}_{1}} \oplus \mathrm{I}_{\mathrm{b}_{2}} \oplus \cdots \oplus \mathrm{I}_{\mathrm{b}_{\mathrm{n}}}\right]\right\}$.

As for the first part of the product we use Theorem 5 to write $\left\{\left[\mathrm{I}_{\mathrm{a}_{\mathrm{p}}}\right]\right\} * \cdots *$ $\left\{\left[\mathrm{I}_{a_{1}}\right]\right\}=\left\{\left[\mathrm{I}_{\mathrm{a}_{1}^{\prime}} \oplus \cdots \oplus \mathrm{I}_{\mathrm{a}_{\mathrm{p}}^{\prime}}\right] \mid\left(\mathrm{a}_{1}^{\prime}, \ldots, \mathrm{a}_{\mathrm{p}}^{\prime}\right) \prec\left(\mathrm{a}_{1}, \ldots, \mathrm{a}_{\mathrm{p}}\right), \sum_{i=1}^{\mathrm{p}} \mathrm{a}_{i}^{\prime}=\sum_{i=1}^{p} a_{i}\right\}$. Hence we have that $\left[\mathrm{I}_{\mathfrak{c}_{1}} \oplus \mathrm{I}_{\mathfrak{c}_{2}} \oplus \cdots \oplus \mathrm{I}_{\mathfrak{c}_{r}}\right] \in\left\{\left[\mathrm{I}_{\mathrm{a}_{\mathrm{p}}}\right]\right\} *\left\{\left[\mathrm{I}_{\mathrm{a}_{\mathfrak{p}-1}}\right]\right\} * \cdots *\left\{\left[\mathrm{I}_{\mathrm{a}_{1}}\right]\right\} *\left\{\left[\mathrm{I}_{\mathrm{b}_{1}}\right]\right\} *$ $\left\{\left[\mathrm{I}_{\mathrm{b}_{2}}\right]\right\} * \cdots *\left\{\left[\mathrm{I}_{\mathrm{b}_{\mathfrak{n}}}\right]\right\}$ if and only if there exists a partition $\boldsymbol{a}^{\prime}=\left(\mathrm{a}_{1}^{\prime}, \ldots, \mathrm{a}_{\mathrm{p}}^{\prime}\right)$ such that $\left|\mathbf{a}^{\prime}\right|=|\mathbf{a}|, \boldsymbol{a}^{\prime} \prec \boldsymbol{a}=\left(\mathrm{a}_{1}, \ldots, \mathrm{a}_{\mathrm{p}}\right)$ and $\left[\mathrm{I}_{\mathrm{c}_{1}} \oplus \cdots \oplus \mathrm{I}_{\mathrm{c}_{\mathrm{r}}}\right] \in\left\{\left[\mathrm{I}_{\mathrm{a}_{1}^{\prime}} \oplus \cdots \oplus \mathrm{I}_{\mathrm{a}_{\mathrm{p}}^{\prime}}\right]\right\} *$ $\left\{\left[\mathrm{I}_{\mathrm{b}_{1}} \oplus \cdots \oplus \mathrm{I}_{\mathrm{b}_{\mathrm{n}}}\right]\right\}$. For the rest of the proof we will work with this equivalent statement.

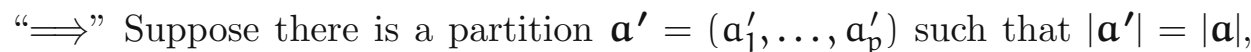
$\boldsymbol{a}^{\prime} \prec \boldsymbol{a}$ and $\left[\mathrm{I}_{\mathrm{c}_{1}} \oplus \cdots \oplus \mathrm{I}_{\mathrm{c}_{\mathrm{r}}}\right] \in\left\{\left[\mathrm{I}_{\mathrm{a}_{1}^{\prime}} \oplus \cdots \oplus \mathrm{I}_{\mathrm{a}_{\mathrm{p}}^{\prime}}\right]\right\} *\left\{\left[\mathrm{I}_{\mathrm{b}_{1}} \oplus \cdots \oplus \mathrm{I}_{\mathrm{b}_{\mathrm{n}}}\right]\right\}$. Using Theorem 4 
we immediately get the equalities $r=n+p$, respectively $\sum_{i=1}^{n+p} c_{i}=\sum_{i=1}^{p} a_{i}+$ $\sum_{i=1}^{n} b_{i}$ and the existence of the strictly increasing functions $\beta:\{1, \ldots, \mathfrak{n}\} \rightarrow$ $\{1, \ldots, n+p\}$ and $\alpha:\{1, \ldots, p\} \rightarrow\{1, \ldots, n+p\}$ with disjoint images such that $b_{i} \geq c_{\beta(i)}$ for $1 \leq i \leq n$ and $\sum_{\beta(i)<\alpha(j)}\left(b_{i}-c_{\beta(i)}\right) \geq \sum_{k=1}^{j}\left(c_{\alpha(k)}-a_{k}^{\prime}\right)$ for all $1 \leq i \leq n$

$j \in\{1, \ldots, p\}$. By reordering the last inequality and using the fact that $\boldsymbol{a}^{\prime} \prec \mathbf{a}$ we obtain

$$
\sum_{\substack{\beta(i)<\alpha(j) \\ 1 \leq i \leq n}} b_{i}+\sum_{k=1}^{j} a_{k} \geq \sum_{\substack{\beta(i)<\alpha(j) \\ 1 \leq i \leq n}} b_{i}+\sum_{k=1}^{j} a_{k}^{\prime} \geq \sum_{k=1}^{j} c_{\alpha(k)}+\sum_{\substack{\beta(i)<\alpha(j) \\ 1 \leq i \leq n}} c_{\beta(i)},
$$

leading to $\sum_{\substack{\beta(i)<\alpha(j) \\ 1 \leq i \leq n}}\left(b_{i}-c_{\beta(i)}\right) \geq \sum_{k=1}^{j}\left(c_{\alpha(k)}-a_{k}\right)$ as desired.

"£" Conversely, suppose that the inequalities (5) and all other conditions from the right-to-left implication are satisfied. If in addition $a_{j} \leq c_{\alpha(j)}$ for $1 \leq \mathfrak{j} \leq \mathbf{p}$, then using Theorem 4 we are done (in this case $\boldsymbol{a}^{\prime}=\mathbf{a}$ ). If $a_{j}>c_{\alpha(j)}$ for some $j \in\{1, \ldots, p\}$, then there exists a raising operator $R$ and $a$ partition $\boldsymbol{a}^{\prime}=\left(a_{1}^{\prime}, \ldots, a_{p}^{\prime}\right)$ such that $\boldsymbol{a}=R \boldsymbol{a}^{\prime}$ and $a_{j}^{\prime} \leq c_{\alpha(j)}$ for $1 \leq j \leq p$ (using the fact that $\sum_{i=1}^{n+p} c_{i}=\sum_{i=1}^{p} a_{i}+\sum_{i=1}^{n} b_{i}$ and $b_{i} \geq c_{\beta(i)}$ for $1 \leq i \leq n$ ). Suppose in addition that $\boldsymbol{a}^{\prime}$ is lexicographically the greatest partition with the mentioned property. Then the inequality

$$
\sum_{\substack{\beta(i)<\alpha(j) \\ 1 \leq i \leq n}}\left(b_{i}-c_{\beta(i)}\right) \geq \sum_{k=1}^{j}\left(c_{\alpha(k)}-a_{k}^{\prime}\right)
$$

is satisfied for all $\boldsymbol{j} \in\{1, \ldots, \mathbf{p}\}$. Since $\boldsymbol{a}=\mathrm{Ra}^{\prime}$, by Corollary 1 we have $\boldsymbol{a}^{\prime} \prec \boldsymbol{a}$ and therefore $\left[\mathrm{I}_{\mathrm{c}_{1}} \oplus \cdots \oplus \mathrm{I}_{\mathrm{c}_{\mathrm{r}}}\right] \in\left\{\left[\mathrm{I}_{\mathrm{a}_{1}^{\prime}} \oplus \cdots \oplus \mathrm{I}_{\mathrm{a}_{\mathrm{p}}^{\prime}}\right]\right\} *\left\{\left[\mathrm{I}_{\mathrm{b}_{1}} \oplus \cdots \oplus \mathrm{I}_{\mathrm{b}_{n}}\right]\right\}$ (since all the conditions from Theorem 4 are fulfilled).

As one can see, all we had to do to obtain the characterization of products of the form $\left\{\left[\mathrm{I}_{\mathfrak{a}_{\mathrm{p}}}\right]\right\} *\left\{\left[\mathrm{I}_{\mathrm{a}_{\mathrm{p}-1}}\right]\right\} * \cdots *\left\{\left[\mathrm{I}_{\mathrm{a}_{1}}\right]\right\} *\left\{\left[\mathrm{I}_{\mathrm{b}_{1}}\right]\right\} *\left\{\left[\mathrm{I}_{\mathrm{b}_{2}}\right]\right\} * \cdots *\left\{\left[\mathrm{I}_{\mathrm{b}_{\mathfrak{n}}}\right]\right\}$ with $0 \leq a_{p} \leq a_{p-1} \leq \cdots \leq a_{1}$ and $b_{1} \geq b_{2} \geq \cdots \geq b_{n} \geq 0$ was a relaxation of Theorem 4 by dropping the condition $a_{j}^{\prime} \leq c_{\alpha(j)}$ for $1 \leq j \leq p$. We can do the very same thing with the explicit version of Theorem 4 (which is Theorem 6 from [19]). We state the following:

Theorem 7 Let $a_{1} \geq \cdots a_{p} \geq 0, b_{1} \geq \cdots \geq b_{n} \geq 0, c_{1} \geq \cdots \geq c_{r} \geq 0$ be decreasing sequences of nonnegative integers and let $B_{j}=\left\{l \in\{0, \ldots, n\} \mid \sum_{k=1}^{l} b_{k}+\right.$ $\left.\sum_{k=1}^{j} a_{k} \geq \sum_{k=1}^{l+j} c_{k}\right\}$ for $1 \leq j \leq p$. Then $\left[\mathrm{I}_{\mathrm{c}_{1}} \oplus \mathrm{I}_{\mathrm{c}_{2}} \oplus \cdots \oplus \mathrm{I}_{\mathrm{c}_{\mathrm{r}}}\right] \in\left\{\left[\mathrm{I}_{\mathrm{a}_{\mathrm{p}}}\right]\right\} *\left\{\left[\mathrm{I}_{\mathrm{a}_{\mathrm{p}-1}}\right]\right\} * \cdots *\left\{\left[\mathrm{I}_{\mathrm{a}_{1}}\right]\right\} *\left\{\left[\mathrm{I}_{\mathrm{b}_{1}}\right]\right\} *\left\{\left[\mathrm{I}_{\mathrm{b}_{2}}\right]\right\} * \cdots *\left\{\left[\mathrm{I}_{\mathrm{b}_{\mathfrak{n}}}\right]\right\}$ 
if and only if $r=p+n, \sum_{i=1}^{r} c_{i}=\sum_{i=1}^{p} a_{i}+\sum_{i=1}^{n} b_{i}, B_{j} \neq \emptyset, b_{i} \geq c_{\beta_{i}}$ for $1 \leq j \leq p$ and $1 \leq i \leq n$, where

$$
\alpha_{j}= \begin{cases}\min \left(B_{1}+1\right), & j=1 \\ \max \left(\alpha_{j-1}+1, \min B_{j}+j\right), & 1<j \leq p\end{cases}
$$

and

$$
\beta_{i}=\left\{\begin{array}{ll}
\min \left(l \in\{1, \ldots, r) \mid l \neq \alpha_{j}, 1 \leq j \leq p\right\}, & i=1 \\
\min \left(l \in\left\{\beta_{i-1}+1, \ldots, r\right\} \mid l \neq \alpha_{j}, 1 \leq j \leq p\right), & 1<i \leq n
\end{array} .\right.
$$

In this case all we had to do was to drop the condition $a_{j} \leq c_{\alpha_{j}}$.

This also leads to a very simple linear-time algorithm (in the number of indecomposables), a slightly modified version of the algorithm given in [19]. Given the preinjective modules $\mathrm{I}_{\mathfrak{c}_{1}} \oplus \mathrm{I}_{\mathrm{c}_{2}} \oplus \cdots \oplus \mathrm{I}_{\mathfrak{c}_{r}}, \mathrm{I}_{\mathrm{a}_{1}}, \ldots, \mathrm{I}_{\mathrm{a}_{\mathrm{p}}}, \mathrm{I}_{\mathrm{b}_{1}}, \ldots, \mathrm{I}_{\mathrm{b}_{\mathfrak{n}}} \in$ $\bmod -k K$ (with $0 \leq a_{p} \leq a_{p-1} \leq \cdots \leq a_{1}$ and $b_{1} \geq b_{2} \geq \cdots \geq b_{n} \geq 0$ ) this is a method one could follow in practice to decide whether $\left[\mathrm{I}_{\mathfrak{c}_{1}} \oplus \mathrm{I}_{\mathrm{c}_{2}} \oplus \cdots \oplus \mathrm{I}_{\mathrm{c}_{\mathrm{r}}}\right] \in$ $\left\{\left[\mathrm{I}_{\mathrm{a}_{\mathrm{p}}}\right]\right\} *\left\{\left[\mathrm{I}_{\mathrm{a}_{\mathrm{p}-1}}\right]\right\} * \cdots *\left\{\left[\mathrm{I}_{\mathrm{a}_{1}}\right]\right\} *\left\{\left[\mathrm{I}_{\mathrm{b}_{1}}\right]\right\} *\left\{\left[\mathrm{I}_{\mathrm{b}_{2}}\right]\right\} * \cdots *\left\{\left[\mathrm{I}_{\mathrm{b}_{\mathrm{n}}}\right]\right\}:$

First check the conditions $r=n+p$ and $\sum_{i=1}^{r} c_{i}=\sum_{i=1}^{p} a_{i}+\sum_{i=1}^{n} b_{i}$. If these are not fulfilled stop with a negative answer, otherwise set the initial values $j=i=k=1$ for the integers used to index elements from the sequences $\left(a_{1}, \ldots, a_{p}\right),\left(b_{1}, \ldots, b_{n}\right)$ respectively $\left(c_{1}, \ldots, c_{r}\right)$. Repeat the following steps for all successive values of $1 \leq k \leq r$ :

1. If $j \leq p$ and $\left(a_{1}+\cdots+a_{j-1}\right)+\left(b_{1}+\cdots+b_{i-1}\right)+a_{j} \geq c_{1}+\cdots+c_{k}$, then increase $\mathrm{j}$ by one.

2. Else, if $\mathrm{i} \leq \mathrm{n}$ and $\mathrm{b}_{\mathrm{i}} \geq \mathrm{c}_{\mathrm{k}}$ and $\left(\mathrm{a}_{1}+\cdots+\mathrm{a}_{\mathrm{j}-1}\right)+\left(\mathrm{b}_{1}+\cdots+\mathrm{b}_{\mathrm{i}-1}\right)+\mathrm{b}_{\mathrm{i}} \geq$ $\mathrm{c}_{1}+\cdots+\mathrm{c}_{\mathrm{k}}$, then increase $i$ by one.

3. If none of the steps above can be carried out than stop with a negative answer.

Finally, if one of the first two steps can be made for $\mathrm{k}=\mathrm{r}$ too, then return a positive answer, i.e. we have $\left[\mathrm{I}_{\mathfrak{c}_{1}} \oplus \mathrm{I}_{\mathfrak{c}_{2}} \oplus \cdots \oplus \mathrm{I}_{\mathfrak{c}_{\mathbf{r}}}\right] \in\left\{\left[\mathrm{I}_{\mathfrak{a}_{p}}\right]\right\} *\left\{\left[\mathrm{I}_{\mathrm{a}_{\mathrm{p}-1}}\right]\right\} * \cdots *$ $\left\{\left[\mathrm{I}_{\mathrm{a}_{1}}\right]\right\} *\left\{\left[\mathrm{I}_{\mathrm{b}_{1}}\right]\right\} *\left\{\left[\mathrm{I}_{\mathrm{b}_{2}}\right]\right\} * \cdots *\left\{\left[\mathrm{I}_{\mathrm{b}_{\mathfrak{n}}}\right]\right\}$.

It is trivial to see that the algorithm is linear in the number of indecomposables (i.e. in $r=n+p$ ), since the only cycle in the algorithm runs at most $r$ times and the partial sums $a_{1}+\cdots+a_{j}, b_{1}+\cdots+b_{i}$ and $c_{1}+\cdots+c_{k}$ can be computed one term at a time at every iteration. 
Finally, we show that the conditions given in Theorem 6 are equivalent to the conditions of the generalized majorization, described in Section 2, establishing a module theoretical background for this notion.

Corollary 2 Let $\mathbf{d}=\left(\mathrm{d}_{1}, \ldots, \mathrm{d}_{\mathrm{x}}\right), \mathbf{a}=\left(\mathrm{a}_{1}, \ldots, \mathrm{a}_{\mathrm{y}}\right)$, and $\mathbf{g}=\left(\mathrm{g}_{1}, \ldots, \mathrm{g}_{\mathrm{x}+\mathrm{y}}\right)$ be partitions. Then

$$
\left[\mathrm{I}_{\mathrm{g}_{1}} \oplus \cdots \oplus \mathrm{I}_{\mathrm{g}_{\mathrm{x}+y}}\right] \in\left\{\left[\mathrm{I}_{\mathrm{a}_{y}}\right]\right\} * \cdots *\left\{\left[\mathrm{I}_{\mathrm{a}_{1}}\right]\right\} *\left\{\left[\mathrm{I}_{\mathrm{d}_{1}}\right]\right\} * \cdots *\left\{\left[\mathrm{I}_{\mathrm{d}_{x}}\right]\right\}
$$

if and only if $\mathbf{g} \prec^{\prime}(\mathbf{d}, \mathbf{a})$, i.e. $\mathbf{g}$ is majorized by $\mathbf{d}$ and $\mathbf{a}$.

Proof. The proof is obviously based on Theorem 6 , hence let us begin by highlighting the equivalent notations: $x=n, y=p, x+y=r, a=\left(a_{1}, \ldots, a_{y}\right)=$ $\left(a_{1}, \ldots, a_{p}\right), \mathbf{d}=\left(d_{1}, \ldots, d_{x}\right)=\left(b_{1}, \ldots, b_{n}\right), \mathbf{g}=\left(g_{1}, \ldots, g_{x+y}\right)=\left(c_{1}, \ldots, c_{r}\right)$, where $n, p, r,\left(a_{1}, \ldots, a_{p}\right),\left(b_{1}, \ldots, b_{n}\right)$ and $\left(c_{1}, \ldots, c_{r}\right)$ are the corresponding variables used in the statement of Theorem 6 .

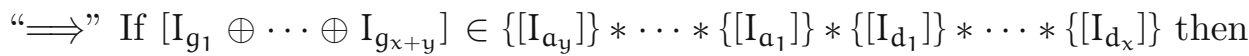
condition (3) from Definition 1 is immediate. We also know that we have a strictly increasing function $\beta:\{1, \ldots, x\} \rightarrow\{1, \ldots, x+y\}$ such that $d_{i} \geq g_{\beta(i)}$ for $1 \leq i \leq x$. Among all these functions let $\beta$ be the function for which the sequence $(\beta(1), \beta(2), \ldots, \beta(x))$ is lexicographically the smallest one with this property. We immediately have for the corresponding function $\alpha:\{1, \ldots, y\} \rightarrow$ $\{1, \ldots, x+y\}$ that $(\alpha(1), \ldots, \alpha(y))=\left(h_{1}, \ldots, h_{y}\right)$ (see Remark 1 at the end of Section 2). By reordering the inequality corresponding to (5) from Theorem 6 we get

$$
\sum_{\substack{\beta(i)<\alpha(j) \\ 1 \leq i \leq x}} d_{i}+\sum_{k=1}^{j} a_{k} \geq \sum_{k=1}^{j} g_{\alpha(k)}+\sum_{\substack{\beta(i)<\alpha(j) \\ 1 \leq i \leq x}} g_{\beta(i)}=\sum_{i=1}^{\alpha(j)} g_{i},
$$

which is equivalent with

$$
\sum_{i=1}^{\alpha(j)} g_{i} \leq \sum_{i=1}^{\alpha(j)-j} d_{i}+\sum_{i=1}^{j} a_{i}
$$

for all $j \in\{1, \ldots, y\}$. Since $(\alpha(1), \ldots, \alpha(y))=\left(h_{1}, \ldots, h_{y}\right)$, this is exactly condition (2) from the definition. Condition (1) also follows easily, since $\beta(i) \leq$ $i+y$ and therefore $d_{i} \geq g_{\beta(i)} \geq g_{i+y}$ for all $i \in\{1, \ldots, x\}$.

"£" Conversely, let $\mathbf{g}$ to be majorized by $\boldsymbol{d}$ and $\boldsymbol{a}$. Set $(\alpha(1), \ldots, \alpha(\mathrm{y}))=$ $\left(h_{1}, \ldots, h_{y}\right)$ and $(\beta(1), \ldots, \beta(x))=\left(h_{1}^{\prime}, \ldots, h_{x}^{\prime}\right)$ as described in Remark 1. Then condition (2) is equivalent with the inequalities (5) from Theorem 6. 
Condition (3) transfers as it is, and we also know that $d_{i} \geq g_{\beta(i)}$ for all $i \in\{1, \ldots, \alpha(y)-y\}$. If $\alpha(y)-y=x$, we are done, otherwise we must have $(\beta(\alpha(y)-y+1), \ldots, \beta(x))=(\alpha(y)+1, \ldots, x+y)$. Considering now condition (1) we can write $d_{i} \geq g_{i+y}=g_{\beta(i)}$ for all $i \in\{\alpha(y)-y+1, \ldots, x\}$, so $d_{i} \geq g_{\beta(i)}$ is fulfilled on the whole range $1 \leq i \leq x$ and the implication now follows by Theorem 6 .

\section{References}

[1] I. Assem, D. Simson, A. Skowroński, Elements of the representation theory of associative algebras, Vol. 1, Techniques of representation theory, London Mathematical Society Student Texts 65, Cambridge University Press, Cambridge, 2006.

[2] M. Auslander, I. Reiten, S. Smalø, Representation theory of Artin algebras, Cambridge Studies in Advanced Mathematics, No. 36, Cambridge University Press, 1995.

[3] M. Dodig, Completion up to a matrix pencil with column minimal indices as the only nontrivial Kronecker invariants, Linear Algebra Appl., 438 (2013), 3155-3173.

[4] M. Dodig, M. Stošić, Combinatorics of column minimal indices and matrix pencil completion problems, SIAM J. Matrix Anal. Appl., 31 (2010), 23182346 .

[5] M. Dodig, M. Stošić, On convexity of polynomial paths and generalized majorizations, Electron. J. Combin., 17 (1) (2010), R61.

[6] M. Dodig, M. Stošić, On properties of the generalized majorization, Electron. J. Linear Algebra, 26 (2013), 471-509.

[7] F. R. Gantmacher, Matrix theory, Vol. 1 and 2, Chelsea, New York, 1974.

[8] Y. Han, Subrepresentations of Kronecker representations, Linear Algebra Appl., 402 (2005), 150-164.

[9] J. J Loiseau, S. Mondié, I. Zaballa, P. Zagalak, Assigning the Kronecker invariants of a matrix pencil by row or column completions, Linear Algebra Appl., 278 (1998), 327-336. 
[10] I. Macdonald, Symmetric Functions and Hall Polynomials, Second edition, Clarendon Press, Oxford, 1995.

[11] M. Reineke, The monoid of families of quiver representations, Proc. Lond. Math. Soc., 84 (2002), 663-685.

[12] C. M. Ringel, Tame algebras and integral quadratic forms, Lecture Notes in Mathematics, No. 1099, Springer-Verlag, Berlin, 1984.

[13] D. Simson, A. Skowroński, Elements of the representation theory of associative algebras, Vol. 2, Tubes and Concealed Algebras of Euclidean Type, London Mathematical Society Student Texts 71, Cambridge University Press, Cambridge, 2007.

[14] Cs. Szántó, Hall numbers and the composition algebra of the Kronecker algebra, Algebr. Represent. Theory, 9 (2006), 465-495.

[15] Cs. Szántó, On the Hall product of preinjective Kronecker modules, Mathematica (Cluj), 48 (71), No. 2 (2006), 203-206.

[16] Cs. Szántó, I. Szöllősi, On preprojective short exact sequences in the Kronecker case, J. Pure Appl. Algebra, 216 (2012), no. 5, 1171-1177.

[17] Cs. Szántó, I. Szöllősi, Preinjective subfactors of preinjective Kronecker modules, arXiv:1309.4710 [math.RT], 13 September 2013, 13 pages.

[18] Cs. Szántó, I. Szöllősi, The terms in the Ringel-Hall product of preinjective Kronecker modules, Period. Math. Hungar., 63 (2) (2011), 75-92.

[19] I. Szöllösi, Computing the extensions of preinjective and preprojective Kronecker modules, J. Algebra, 408 (2014) 205-221.

[20] I. Szöllősi, The extension monoid product of preinjective Kronecker modules, Mathematica (Cluj), 55 (78), No. 1 (2013), 75-88.

Received: 9 April 2014 